\title{
The Absorption Spectra of Krypton and Xenon in the Wavelength Range 330-600 A
}

\author{
K. Codling* and R. P. Madden \\ Institute for Basic Standards, National Bureau of Standards, Washington, D.C. 20234
}

(September 20, 1971)

\begin{abstract}
A total of 153 krypton resonances in the spectral region 330-500 A and 253 xenon resonances in the spectral region 375-600 $\AA$ are reported. A detailed listing of the resonances is given, with wavelength and line shape information. The analysis of the spectra is very incomplete and will require detailed theoretical calculation to significantly improve it. $\mathrm{In} \mathrm{Kr}, 45$ resonances and in $\mathrm{Xe}, 56$ resonances have been grouped into probable Rydberg series, for which classifications are suggested. The resonances are due, in the main, to either the excitation of the inner subshell " $s$ " electron $\left(s^{2} p^{6} \rightarrow s p^{6} n p\right)$ or to the excitation of two of the outer " $p$ " electrons simultaneously $\left(s^{2} p^{6} \rightarrow s^{2} p^{4} n l n^{\prime} l^{\prime}\right)$. These highlying excited states autoionize, resulting in resonances with window-, asymmetric-, or absorption-type profiles. Where possible, comparisons are made with previous work.
\end{abstract}

Key words: Autoionization; far ultraviolet; krypton; photoionization; resonances; xenon.

\section{Introduction}

This communication is intended as the final report on the absorption spectra of the noble gases He through Xe, observed in the wavelength range $80-600 \AA$. Earlier papers have dealt with the discovery and analysis (in some cases tentative) of the structure in the photoionization continuua of $\mathrm{He}[1],{ }^{1} \mathrm{Ne}[2]$, and $\mathrm{Ar}[3]$. We present here the spectra of $\mathrm{Kr}$ and $\mathrm{Xe}$ in the wavelength range $330-600 \AA$. Two previous publications have described the structure observed between 80 and $200 \AA$, due to the excitation of a single innershell $d$ electron[4] and also the simultaneous excitation of an outer-shell $p$ and an inner-shell $d$ electron[5]. (The excitation of the outer $p$ electron had been observed many years before [6].)

In the wavelength range $400-500 \AA$, Samson [7] observed two distinct resonances in the photoionization continuum of $\mathrm{Kr}$ using a many-lined spark discharge as a background source. Subsequently, many more autoionizing states in both $\mathrm{Kr}$ and $\mathrm{Xe}$ between 380 and $600 \AA$ were reported [8] and recently, highlights of the present, more detailed experimental observations of the $\mathrm{Kr}$ and $\mathrm{Xe}$ spectra have been published [9]. In an accompanying paper [10], absolute cross-section measurements were made in the region of some of the more prominent resonances.

The following analysis of the $\mathrm{Kr}$ and Xe spectra is necessarily far from complete. The breakdown of strict $L-S$ coupling, evident throughout the Ar spectrum, is even more pronounced in $\mathrm{Kr}$ and $\mathrm{Xe}$. Consequently, considerable theoretical work is re-

\footnotetext{
*Present Address: University of Reading, Physics Department, Whiteknights Park Reading, Berks, England.

${ }^{1}$ Figures in brackets indicate the literature references at the end of this paper.
}

quired, of an $a b$ initio type, before a reasonable analysis of the $\mathrm{Kr}$ and $\mathrm{Xe}$ spectra can be made. In the hope of stimulating such calculations on noble gas atoms, we present here final numbers on wavelengths of the resonances observed, along with a rough guide as to the profiles of some of the resonances. The accompanying spectra and densitometer traces together with the published resonance profiles [10] may be helpful in comparing theoretical predictions with the experimental observations, at least for the low-lying levels.

\section{Experimental Procedure}

The spectra shown in figure 1 were taken using a three-meter grazing-incidence spectrograph [11]. The grating of 600 lines per $\mathrm{mm}$ and a $0.01 \mathrm{~mm}$ spectrograph entrance slit yielded a resolution of $0.06 \mathrm{~A}$. The source of continuum was provided by the radiation emitted by the NBS-SURF (Synchrotron Ultraviolet Radiation Facility). For this synchrotron the radiated power per unit wavelength peaks at $335 \AA$. Spectra were photographed from $80-600 \AA$ with a grating blazed at $200 \AA$. The noble gas filled the spectrograph at pressures typically in the range of 0.01 to 0.10 torr, with absorption path lengths between entrance slit and plate of the order of $1 \mathrm{~m}$. Since the synchrotron continuum is free from overlying emission and absorption lines, any features observed were due to the gas itself.

The spectra were photographed on Eastman SWR plates. ${ }^{2}$ W avelength calibration was obtained by super-

${ }^{2} \mathrm{~A}$ commercial material is identified in this paper in order to adequately specify the experimental procedure. Such identification does not imply recommendation or endorsement by the National Bureau of Standards. 

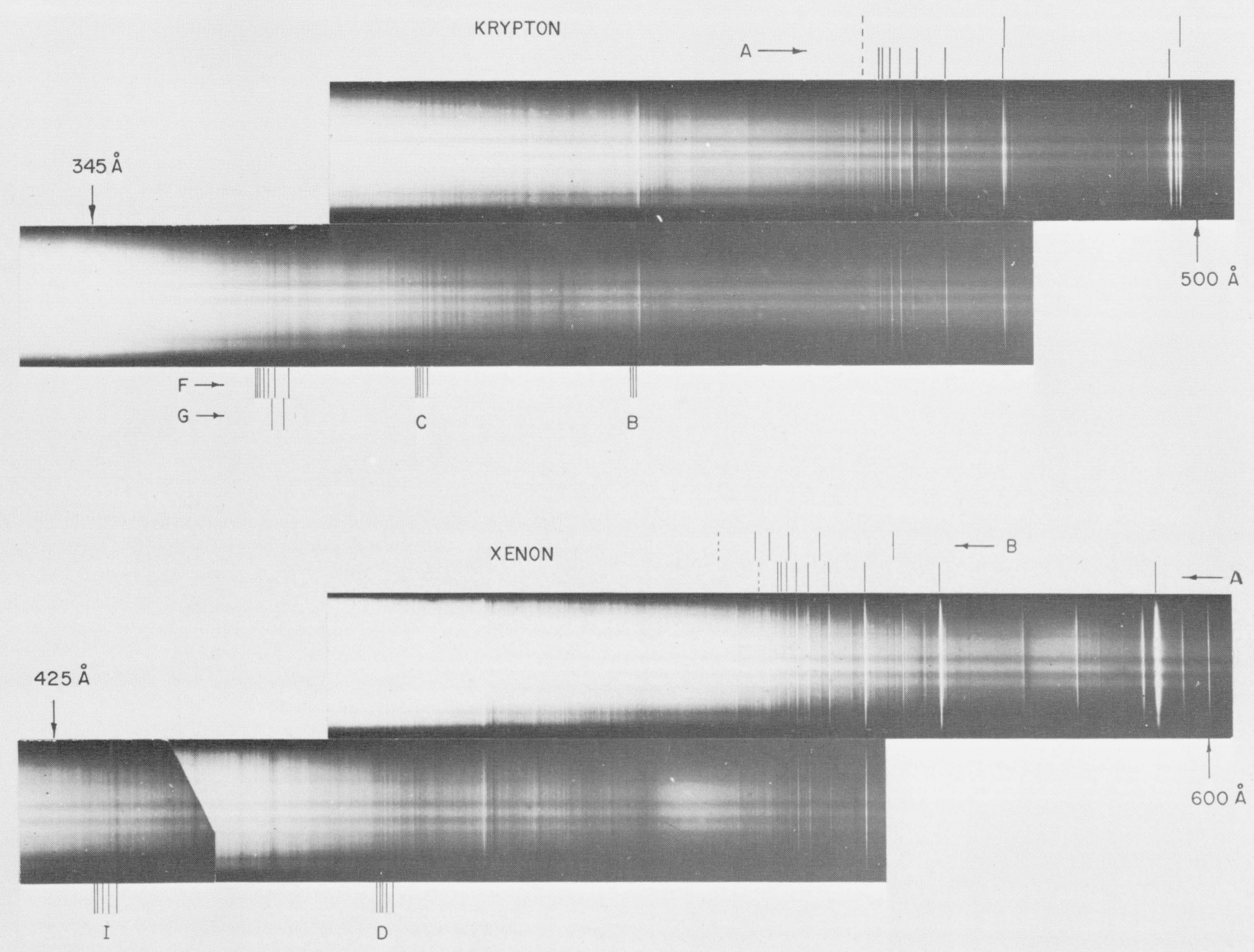

FIGURE 1. Upper Spectrum. The absorption spectrum of $\mathrm{Kr}$ between 345 and $500 \AA$, taken at two different pressures to accentuate the various regions of the spectrum.

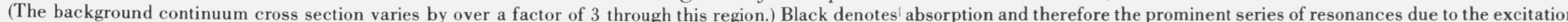

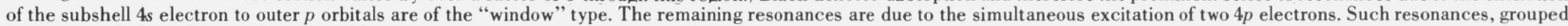
in series B, C, F, and $\mathrm{G}$, are listed in table 3 .

Lower Spectrum. The absorption spectrum of Xe between 425 and $600 \AA$, taken at three different pressures.

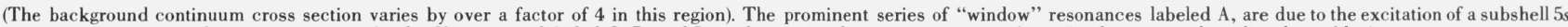
electron to outer $p$ orbitals. Short series labeled C, D, and I are due to simultaneous excitation of two $5 p$ electrons and are listed in table 4 .

imposing the absorption spectrum of $\mathrm{He}$ on the $\mathrm{Kr}$ and $\mathrm{Xe}$ spectra and by using the previously determined fact that wavelengths could be reliably predicted, by application of the grating equation, to an accuracy of at least $0.01 \AA$. As an additional check, where long Rydberg series existed, use was made of the fact that such series converge in the limit to a well established state of $\mathrm{Kr}$ II or Xe II. In the cases where only small perturbations exist good quantum defects were obtained using known limits and the measured wavelengths of the Rydberg series lines.

\section{Classification of Spectra}

The ground state of $\mathrm{Kr}$ is $4 s^{2} 4 p^{6}{ }^{1} \mathrm{~S}_{0}$, that of $\mathrm{Xe}$ $5 s^{2} 5 p^{6}{ }^{1} \mathrm{~S}_{0}$. The ionization potentials of $\mathrm{Kr}$ and $\mathrm{Xe}$ are 14.0 and $12.1 \mathrm{eV}$ respectively. Thus, when photons of an energy between 20 and $40 \mathrm{eV}$ are incident upon these gases, the basic process observed is photoionization. However, as well as exciting an outer $p$ electron to continuum $\epsilon s$ and $\epsilon d$ states, it is possible to excite a subshell $s$ electron to $p$-type orbitals or excite two electrons simultaneously. In the latter case, either two outer $p$ electrons or both an outer $p$ and a subshell $s$ electron can be excited.

Thus, in $\mathrm{Kr}$ for example, one may excite to configurations of the type: $4 s 4 p^{6} n p$ and $4 s^{2} 4 p^{4} n l n^{\prime} l^{\prime}$ where $l l^{\prime}=s p, p d, s f, d f$. Transitions of the type $4 s 4 p^{5} n l n^{\prime} l^{\prime}$ where $l l^{\prime}=s s, p p, s d$ etc., have not been positively identified in $\mathrm{Kr}$ and $\mathrm{Xe}$, although unidentified resonances exist in the appropriate energy region, and the existence of similar excitations has been fairly well established in Ar [3].

The total line list of 153 resonances in $\mathrm{Kr}$ and 253 resonances in $\mathrm{Xe}$ are listed in terms of their wave- 
length, wavenumber and resonance type in tables 1 and 2. By resonance type we mean the following. When a high-lying discrete state such as the $4 s 4 p^{6} 5 p$ state of $\mathrm{Kr}$ is excited by photoabsorption, the lifetime of this excited state is extremely short $\left(\sim 10^{-13} \mathrm{~s}\right)$. The state decays into the adjacent continuum by autoionization, with the ejection of an energetic electron, the ion being left in its ground or excited state. The interaction of a discrete state with the continuum of states of similar energy and parity has been dealt with by Fano and Cooper [12]. The resonances in the continuum which result from this interaction may be broad, and can have a variety of profiles.

TABLE 1. Code number, wavelength $(\lambda)$, wave number $(\nu)$, and profile type for all observed $\mathrm{Kr}$ resonances, listed in order of decreasing wavelength

\begin{tabular}{|c|c|c|c|c|c|c|c|}
\hline Number & Wavelength $(\AA)$ & $\begin{array}{l}\text { Wave number } \\
\qquad\left(\mathrm{cm}^{-1}\right)\end{array}$ & Comment $^{\mathrm{a}}$ & Number & W avelength $(\AA)$ & $\begin{array}{l}\text { Wave number } \\
\quad\left(\mathrm{cm}^{-1}\right)\end{array}$ & Comment $^{\mathrm{a}}$ \\
\hline 1 & b $501.23 \pm .05$ & 199509 & $-s x$ & 52 & $421.56 \pm .03$ & 237214 & $-s x$ \\
\hline 2 & $498.83 \pm .05$ & 200469 & $-d w$ & 53 & $421.23 \pm .03$ & 237400 & $-s x$ \\
\hline 3 & $c * 497.50 \pm .05$ & 201005 & $+d w$ & 54 & $420.90 \pm .05$ & 237586 & $-s x$ \\
\hline 4 & $496.90 \pm .05$ & 201248 & $d w$ & 55 & $420.36 \pm .05$ & 237891 & $-d a$ \\
\hline 5 & $* 496.07 \pm .05$ & 201584 & $-d w$ & 56 & $419.96 \pm .05$ & 238118 & $s a$ \\
\hline 6 & $492.52 \pm .05$ & 203037 & $-s w$ & 57 & $419.74 \pm .05$ & 238243 & $s a$ \\
\hline 7 & $488.71 \pm .05$ & 204620 & $-d x$ & 58 & $419.51 \pm .05$ & 238373 & $s a$ \\
\hline 8 & $488.06 \pm .05$ & 204893 & $-d x$ & 59 & $419.10 \pm .10$ & 238607 & $d a$ \\
\hline 9 & $472.26 \pm .03$ & 211748 & $+s w$ & 60 & $418.83 \pm .03$ & 238760 & $s w$ \\
\hline 10 & $* 471.48 \pm .03$ & 212098 & $+s w$ & 61 & $418.70 \pm .03$ & 238834 & $s w$ \\
\hline 11 & $* 471.23 \pm .03$ & 212211 & $-s w$ & 62 & $418.33 \pm .05$ & 239046 & $s a$ \\
\hline 12 & $469.08 \pm .03$ & 213183 & $-s x$ & 63 & $* 417.96 \pm .03$ & 239257 & $s a$ \\
\hline 13 & $464.70 \pm .10$ & 215193 & $\begin{array}{l}-s x \\
-s w\end{array}$ & 64 & $* 417.62 \pm .03$ & 239452 & $s a$ \\
\hline 14 & $* 462.71 \pm .03$ & 216118 & $+s w$ & 65 & $* 417.39 \pm .05$ & 239584 & $s a$ \\
\hline 15 & $461.83 \pm .03$ & 216530 & $-s x$ & 66 & $* 417.22 \pm .05$ & 239682 & $s a$ \\
\hline 16 & $460.45 \pm .03$ & 217179 & sa & 67 & $416.76 \pm .05$ & 239946 & $s w$ \\
\hline 17 & $459.91 \pm .03$ & 217434 & $-s a$ & 68 & $416.01 \pm .10$ & 240379 & $d w$ \\
\hline 18 & $* 458.69 \pm .03$ & 218012 & $+s w$ & 69 & $\mathrm{~d} 414.78 \pm .10$ & 241092 & $s w$ \\
\hline 19 & $457.86 \pm .03$ & 218407 & $-s w$ & 70 & $413.58 \pm .10$ & 241791 & $s w$ \\
\hline 20 & $457.51 \pm .03$ & 218574 & sa & 71 & $412.79 \pm .05$ & 242254 & $+s w$ \\
\hline 21 & $456.67 \pm .03$ & 218976 & sa & 72 & $412.47 \pm .05$ & 242442 & $s w$ \\
\hline 22 & $* 456.12 \pm .03$ & 219241 & $s w$ & 73 & $409.70 \pm .2$ & 244081 & $d d a$ \\
\hline 23 & $455.29 \pm .03$ & 219640 & $-s x$ & 74 & $406.85 \pm .10$ & 245791 & $d a$ \\
\hline 24 & $* 454.73 \pm .03$ & 219911 & $s w$ & 75 & $405.30 \pm .10$ & 246731 & $d a$ \\
\hline 25 & $454.01 \pm .03$ & 220259 & sa & 76 & $403.34 \pm .05$ & 247930 & $d a$ \\
\hline 26 & $* 453.73 \pm .03$ & 220395 & $s w$ & 77 & $401.50 \pm .2$ & 249070 & $d d a$ \\
\hline 27 & $* 453.15 \pm .03$ & 220677 & $s w$ & 78 & $400.33 \pm .07$ & 249794 & $d a$ \\
\hline 28 & $* 452.68 \pm .03$ & 220907 & $s w$ & 79 & $399.75 \pm .07$ & 250156 & $d a$ \\
\hline 29 & $* 452.34 \pm .03$ & 221073 & $\begin{array}{l}s w \\
s w\end{array}$ & 80 & $399.30 \pm .10$ & 250438 & $d a$ \\
\hline 30 & $* 452.07 \pm .03$ & 221205 & $s w$ & 81 & $396.77 \pm .10$ & 252035 & $-d w$ \\
\hline 31 & $451.98 \pm .03$ & 221249 & $\begin{array}{l}s w \\
s w\end{array}$ & 82 & $394.24 \pm .07$ & 253653 & $d a$ \\
\hline 32 & $* 451.86 \pm .03$ & 221307 & $\begin{array}{l}s w \\
s w\end{array}$ & 83 & $393.50 \pm .07$ & 254130 & $d a$ \\
\hline 33 & $* 451.69 \pm .03$ & 221391 & $\begin{array}{l}s w \\
s w\end{array}$ & 84 & $392.70 \pm .07$ & 254647 & $d a$ \\
\hline 34 & $* 451.56 \pm .03$ & 221455 & $s w$ & 85 & $391.83 \pm .05$ & 255213 & $d a$ \\
\hline 35 & $* 451.45 \pm .03$ & 221508 & $\begin{array}{l}s w \\
s w\end{array}$ & 86 & $* 391.44 \pm .05$ & 255467 & sa \\
\hline 36 & $450.16 \pm .03$ & 222143 & $\begin{array}{r}s w \\
-s w\end{array}$ & 87 & $* 390.49 \pm .05$ & 256088 & $d a$ \\
\hline 37 & $449.93 \pm .03$ & 222257 & $\begin{array}{l}-s w \\
+s w\end{array}$ & 88 & $390.04 \pm .05$ & 256384 & $s a$ \\
\hline 38 & $449.22 \pm .03$ & 222608 & $\begin{array}{l}+s w \\
-s w\end{array}$ & 89 & $* 389.48 \pm .05$ & 256753 & $d a$ \\
\hline 39 & $448.29 \pm .03$ & 223070 & $\begin{array}{l}-s w \\
+s w\end{array}$ & 90 & $* 388.84 \pm .05$ & 257175 & $d a$ \\
\hline 40 & $446.54 \pm .05$ & 223944 & $\begin{array}{l}+s w \\
-s x\end{array}$ & 91 & $* 388.37 \pm .05$ & 257486 & $s a$ \\
\hline 41 & $444.02 \pm .03$ & 225215 & $\begin{array}{l}-s x \\
+s x\end{array}$ & 92 & $* 388.05 \pm .05$ & 257699 & $s a$ \\
\hline 42 & $439.00 \pm .2$ & $\begin{array}{l}225215 \\
227790\end{array}$ & $+s x$ & 93 & $* 387.79 \pm .05$ & 257872 & $s a$ \\
\hline 43 & $434.30 \pm .05$ & 230256 & $\begin{array}{r}d w \\
-d w\end{array}$ & 94 & $* 387.60 \pm .05$ & 257998 & sa \\
\hline 44 & $433.98 \pm .05$ & $\begin{array}{l}230250 \\
230425\end{array}$ & $-d w$ & 95 & $387.45 \pm .05$ & 258098 & $s a$ \\
\hline 45 & $427.15 \pm .03$ & $\begin{array}{l}230425 \\
234110\end{array}$ & $s w$ & 96 & $* 387.08 \pm .05$ & 258345 & $-d a$ \\
\hline 46 & $426.53 \pm .05$ & & $s a$ & 97 & $386.26 \pm .05$ & 258893 & $-d a$ \\
\hline 47 & $425.33+.07$ & & $-d a$ & 98 & $385.45 \pm .07$ & 259437 & $s a$ \\
\hline 48 & $425.35=.06$ & 235112 & $d a$ & 99 & $* 385.03 \pm .05$ & 259720 & $-s w$ \\
\hline 48 & $424.05 \pm .05$ & 235821 & $d w$ & 100 & $384.38 \pm .05$ & 260159 & $s a$ \\
\hline 49 & $423.61 \pm .05$ & 236066 & $s w$ & 101 & ${ }^{*} 384.03 \pm .05$ & 260396 & $-s w$ \\
\hline 50 & $423.17 \pm .07$ & 236312 & $d w$ & 102 & $383.47 \pm .05$ & 260777 & $s a$ \\
\hline 51 & $422.58 \pm .03$ & 236642 & $s a$ & 103 & $383.22 \pm .05$ & 260947 & $s a$ \\
\hline
\end{tabular}


TABLE 1. Code number, wavelength $(\lambda)$, wave number $(\nu)$, and profile type for all observed $\mathrm{Kr}$ resonances, listed in order of decreasing wavelength-Continued

\begin{tabular}{|c|c|c|c|c|c|c|c|}
\hline Number & Wavelength $(\AA)$ & $\begin{array}{c}\text { Wave: number } \\
\left(\mathrm{cm}^{-1}\right)\end{array}$ & Comment $^{a}$ & Number & Wavelength $(\AA)$ & $\begin{array}{l}\text { Wave number } \\
\quad\left(\mathrm{cm}^{-1}\right)\end{array}$ & Comment $^{a}$ \\
\hline 104 & $382.95 \pm .05$ & 261131 & $s a$ & 129 & $* 367.03 \pm .05$ & 272457 & $+s a$ \\
\hline 105 & ${ }^{\mathrm{d}} 382.45 \pm .05$ & 261472 & $d a$ & 130 & $* 366.70 \pm .05$ & 272702 & $+s a$ \\
\hline 106 & $382.07 \pm .05$ & 261732 & $s a$ & 131 & $* 366.49 \pm .05$ & 272859 & $+s a$ \\
\hline 107 & $381.87 \pm .05$ & 261869 & sa & 132 & $365.50 \pm .2$ & 273600 & $d d a$ \\
\hline 108 & $381.51 \pm .05$ & 262116 & $s a$ & 133 & $363.80 \pm .2$ & 274880 & $d d a$ \\
\hline 109 & $381.31 \pm .05$ & 262254 & $s a$ & 134 & $362.70 \pm .2$ & 275710 & $s a$ \\
\hline 110 & $380.80 \pm .05$ & 262605 & $s a$ & 135 & $362.30 \pm .2$ & 276010 & $s a$ \\
\hline 111 & $379.30 \pm .2$ & 263640 & $d d a$ & 136 & $361.90 \pm .2$ & 276320 & $s a$ \\
\hline 112 & $* 378.97 \pm .05$ & 263873 & $s a$ & 137 & $359.60 \pm .2$ & 278090 & $s a$ \\
\hline 113 & ${ }^{*} 378.65 \pm .05$ & 264096 & $s a$ & 138 & $358.20 \pm .2$ & 279170 & $s a$ \\
\hline 114 & $* 378.43 \pm .05$ & 264250 & $s a$ & 139 & $357.30 \pm .2$ & 279880 & $s a$ \\
\hline 115 & $* 378.23 \pm .05$ & 264389 & $s a$ & 140 & $356.50 \pm .2$ & 280500 & $s a$ \\
\hline 116 & $375.03 \pm .2$ & 265390 & $d d a$ & 141 & $d 355.60 \pm .2$ & 281210 & $d a$ \\
\hline 117 & $5.03 \pm .05$ & 266645 & $s a$ & 142 & $354.80 \pm .2$ & 281850 & $d a$ \\
\hline 118 & $374.66 \pm .05$ & 266909 & $s a$ & 143 & $354.40 \pm .2$ & 282170 & $d a$ \\
\hline 119 & $374.20 \pm .05$ & 267237 & $s a$ & 144 & $350.30 \pm .2$ & 285470 & $d a$ \\
\hline 120 & $373.00 \pm .2$ & 268100 & $d d a$ & 145 & $349.30 \pm .2$ & 286290 & $d a$ \\
\hline 121 & $371.86 \pm .05$ & 268918 & $s a$ & 146 & $348.30 \pm .2$ & 287110 & $d a$ \\
\hline 122 & $371.37 \pm .05$ & 269273 & $s a$ & 147 & $347.00 \pm .2$ & 288180 & $d a$ \\
\hline 123 & $* 370.87 \pm .05$ & 269636 & $s a$ & 148 & $345.10 \pm .2$ & 289770 & $d d a$ \\
\hline 124 & $* 370.13 \pm .05$ & 270175 & $s w$ & 149 & $343.50 \pm .2$ & 291120 & $d a$ \\
\hline 125 & $* 369.13 \pm .05$ & 270907 & $s a$ & 150 & $342.30 \pm .2$ & 292140 & $s a$ \\
\hline 126 & $* 368.60 \pm .05$ & 271297 & $s w$ & 151 & $341.80 \pm .2$ & 292570 & $s a$ \\
\hline 127 & $* 368.14 \pm .05$ & 271636 & $s a$ & 152 & $341.10 \pm .2$ & 293170 & $s a$ \\
\hline 128 & $* 367.56 \pm .05$ & 272064 & $+s a$ & 153 & $337.40 \pm .3$ & 296380 & $d a$ \\
\hline
\end{tabular}

${ }^{a}$ Sign plus (+) or minus (-) represents the sign of $q$ in the Fano [12] representation of noninteracting resonances. The letters s, d, dd, give a very rough idea of the sharpness or diffuseness of lines on a photographic plate. Naturally, this apparent width varies with gas pressure. The letters $x, w$ or $a$ indicate specifically if the resonance has been measured at $(x)$ the point of highest rate of change of plate density, $(w)$ the point of maximum plate density, or $(a)$ the point of minimum plate density (maximum absorption); e.g., sw means a sharp resonance of low unknown (q) measured at the peak transmission point.

${ }^{b}$ Errors shown represent the estimated absolute uncertainty. In the case of $\mathrm{Kr}$ the relative accuracies are of the order of $0.02 \AA$ better than the estimated absolute uncertainty.

${ }^{\mathrm{c}}$ Resonances denoted by an asterisk $(*)$ are listed in table 3 as possible members of Rydberg series.

d Possible double resonance.

TABLE 2. Code number, wavelength $(\lambda)$, wave number $(\nu)$, and profile type for all observed Xe resonances, listed in order of decreasing wavelength

\begin{tabular}{|c|c|c|c|c|c|c|c|}
\hline Number & Wavelength $(\AA)$ & $\begin{array}{l}\text { Wave number } \\
\qquad\left(\mathrm{cm}^{-1}\right)\end{array}$ & Comment $^{\mathrm{a}}$ & Number & Wavelength $(\AA)$ & $\begin{array}{l}\text { Wave number } \\
\qquad\left(\mathrm{cm}^{-1}\right)\end{array}$ & Comment $^{\mathrm{a}}$ \\
\hline 1 & ${ }^{b} 599.99 \pm .05$ & 166669 & $s w$ & 17 & $556.51 \pm .02$ & 179691 & $+s x$ \\
\hline 2 & $595.93 \pm .03$ & 167805 & $-s w$ & 18 & $555.52 \pm .02$ & 180012 & $-s w$ \\
\hline 3 & $c * 591.77 \pm .03$ & 168985 & $+d w$ & 19 & $555.28 \pm .02$ & 180089 & $s w$ \\
\hline 4 & $589.54 \pm .02$ & 169624 & $-s w$ & 20 & $552.31 \pm .02$ & 181058 & $+s x$ \\
\hline 5 & $586.29 \pm .02$ & 170564 & $-s w$ & 21 & $552.00 \pm .02$ & 181159 & $s w$ \\
\hline 6 & $582.74 \pm .02$ & 171603 & $-s x$ & 22 & $* 550.76 \pm .02$ & 181567 & $+s x$ \\
\hline 7 & $581.11 \pm .02$ & 172084 & $s w$ & 23 & $549.60 \pm .02$ & 181951 & $s a$ \\
\hline 8 & $579.98 \pm .02$ & 172420 & $s w$ & 24 & $549.19 \pm .05$ & 182086 & $s a$ \\
\hline 9 & $579.16 \pm .02$ & 172664 & $-s w$ & 25 & $548.14 \pm .02$ & 182435 & sa \\
\hline 10 & $570.79 \pm .02$ & 175196 & $s w$ & 26 & $547.79 \pm .02$ & 182552 & $s a$ \\
\hline 11 & $570.47 \pm .05$ & 175294 & $s w$ & 27 & $546.88 \pm .02$ & 182855 & $s a$ \\
\hline 12 & $562.39 \pm .02$ & 177813 & $s a$ & 28 & $* 546.08 \pm .02$ & 183123 & $+s w$ \\
\hline 13 & $560.64 \pm .05$ & 178368 & $-s a$ & 29 & $545.26 \pm .02$ & 183399 & $s w$ \\
\hline 14 & $560.44 \pm .05$ & 178431 & $+s a$ & 30 & $544.95 \pm .05$ & 183503 & $s a$ \\
\hline 15 & $558.78 \pm .02$ & 178961 & $-s x$ & 31 & $* 544.18 \pm .02$ & 183763 & $-s x$ \\
\hline 16 & $* 557.83 \pm .02$ & 179266 & $+s w$ & 32 & $543.89 \pm .02$ & 183861 & $+s w$ \\
\hline
\end{tabular}


TABLE 2. Code number, wavelength $(\lambda)$, wave number $(\nu)$, and profile type for all observed $\mathrm{Xe}$ resonances listed in order of decreasing wavelength-Continued

\begin{tabular}{|c|c|c|c|c|c|c|c|}
\hline Number & Wavelength $(\AA)$ & $\begin{array}{l}\text { Wave number } \\
\quad\left(\mathrm{cm}^{-1}\right)\end{array}$ & Comment $^{\mathrm{a}}$ & Number & Wavelength $(\AA)$ & $\begin{array}{l}\text { Wave number } \\
\left(\mathrm{cm}^{-1}\right)\end{array}$ & Comment $^{\mathrm{a}}$ \\
\hline 33 & $543.49 \pm .05$ & 183996 & $s w$ & 95 & $499.74 \pm .03$ & 200104 & $-s w$ \\
\hline 34 & $541.95 \pm .02$ & 184519 & $s a$ & 96 & $498.54 \pm .03$ & 200586 & $s a$ \\
\hline 35 & ${ }^{d} 541.46 \pm .05$ & 184686 & $s a$ & 97 & $497.33 \pm .05$ & 201074 & $s w$ \\
\hline 36 & $540.62 \pm .02$ & 184973 & $s w$ & 98 & $497.06 \pm .05$ & 201183 & $+s x$ \\
\hline 37 & $* 540.54 \pm .02$ & 185000 & $s w$ & 99 & $496.11 \pm .05$ & 201568 & $d a$ \\
\hline 38 & $* 539.33 \pm .02$ & 185415 & $-s x$ & 100 & $495.69 \pm .10$ & 201739 & $s a$ \\
\hline 39 & $537.75 \pm .02$ & 185960 & $s a$ & 101 & $495.42 \pm .05$ & 201849 & $s w$ \\
\hline 40 & $* 537.46 \pm .02$ & 186060 & $s w$ & 102 & $495.15 \pm .05$ & 201959 & $s w$ \\
\hline 41 & $537.38 \pm .02$ & 186088 & $s w$ & 103 & $495.02 \pm .05$ & 202012 & $s a$ \\
\hline 42 & $537.22 \pm .02$ & 186143 & $s w$ & 104 & $494.39 \pm .05$ & 202269 & $-s w$ \\
\hline 43 & $536.83 \pm .02$ & 186279 & $-s w$ & 105 & $493.80 \pm .05$ & 202511 & $-s a$ \\
\hline 44 & $535.95 \pm .02$ & 186585 & $-s a$ & 106 & $493.42 \pm .05$ & 202667 & $-s a$ \\
\hline 45 & $* 535.55 \pm .02$ & 186724 & $s w$ & 107 & $492.91 \pm .03$ & 202877 & $s a$ \\
\hline 46 & $535.13 \pm .02$ & 186870 & $-s x$ & 108 & $492.10 \pm .2$ & 203210 & $d d a$ \\
\hline 47 & $* 534.58 \pm .02$ & 187063 & $-s x$ & 109 & $491.22 \pm .03$ & 203575 & $-s w$ \\
\hline 48 & $* 534.27 \pm .02$ & 187171 & $s w$ & 110 & $490.20 \pm .05$ & 203998 & $-s x$ \\
\hline 49 & $d * 533.34 \pm .05$ & 187498 & $s w$ & 111 & $489.93 \pm .05$ & 204111 & $s a$ \\
\hline 50 & $533.00 \pm .02$ & 187617 & $-s w$ & 112 & $489.66 \pm .03$ & 204223 & $s a$ \\
\hline 51 & $* 532.76 \pm .02$ & 187702 & $s w$ & 113 & $489.19 \pm .03$ & 204420 & $d a$ \\
\hline 52 & $532.06 \pm .02$ & 187949 & $-s w$ & 114 & $488.91 \pm .03$ & 204537 & $s a$ \\
\hline 53 & $531.83 \pm .02$ & 188030 & $-s w$ & 115 & $488.59 \pm .03$ & 204671 & $+s a$ \\
\hline 54 & $* 531.37 \pm .02$ & 188193 & $-s w$ & 116 & $488.23 \pm .03$ & 204821 & $+s a$ \\
\hline 55 & $* 531.21 \pm .02$ & 188249 & $s w$ & 117 & $487.93 \pm .03$ & 204947 & $s a$ \\
\hline 56 & $531.04 \pm .02$ & 188310 & $s w$ & 118 & $487.79 \pm .03$ & 205006 & $s w$ \\
\hline 57 & $* 530.93 \pm .02$ & 188349 & $s w$ & 119 & $487.68 \pm .03$ & 205052 & $s w$ \\
\hline 58 & $* 530.83 \pm .03$ & 188384 & $s w$ & 120 & $487.03 \pm .05$ & 205326 & $s w$ \\
\hline 59 & $* 530.74 \pm .03$ & 188416 & $s w$ & 121 & $486.71 \pm .05$ & 205461 & $s w$ \\
\hline 60 & $530.10 \pm .02$ & 188644 & $-s w$ & 122 & $485.55 \pm .03$ & 205952 & $+s w$ \\
\hline 61 & $529.94 \pm .03$ & 188701 & $s w$ & 123 & $485.25 \pm .05$ & 206079 & $-s w$ \\
\hline 62 & $* 529.34 \pm .02$ & 188914 & $s w$ & 124 & $483.12 \pm .05$ & 206988 & $+s a$ \\
\hline 63 & $528.93 \pm .03$ & 189061 & $s a$ & 125 & $482.20 \pm .05$ & 207383 & $s a$ \\
\hline 64 & $* 528.42 \pm .03$ & 189243 & $+s a$ & 126 & $481.28 \pm .05$ & 207779 & $s w$ \\
\hline 65 & $* 528.05 \pm .05$ & 189376 & $+s w$ & 127 & $480.47 \pm .05$ & 208130 & $-s a$ \\
\hline 66 & $526.35 \pm .02$ & 189988 & $-t s w$ & 128 & $478.93 \pm .10$ & 208799 & $d a$ \\
\hline 67 & $526.15 \pm .02$ & 190060 & $s w$ & 129 & $478.30 \pm .05$ & 209074 & $d a$ \\
\hline 68 & $525.86 \pm .02$ & 190165 & $s w$ & 130 & $477.51 \pm .05$ & 209420 & $d a$ \\
\hline 69 & $* 525.77 \pm .02$ & 190197 & $s w$ & 131 & $477.12 \pm .05$ & 209591 & $s w$ \\
\hline 70 & $525.27 \pm .03$ & 190378 & $-\beta s w$ & 132 & $476.77 \pm .05$ & 209745 & $s w$ \\
\hline 71 & $521.97 \pm .05$ & 191582 & $s w$ & 133 & $476.00 \pm .3$ & 210080 & $d d a$ \\
\hline 72 & $521.81 \pm .05$ & 191641 & $s w$ & 134 & $475.19 \pm .05$ & 210442 & $s w$ \\
\hline 73 & $521.54 \pm .10$ & 191740 & $d a$ & 135 & $* 474.36 \pm .05$ & 210810 & $d a$ \\
\hline 74 & $518.88 \pm .10$ & 192723 & sa & 136 & $* 473.42 \pm .05$ & 211229 & $d a$ \\
\hline 75 & $517.24 \pm .10$ & 193334 & $d a$ & 137 & $* 472.83 \pm .05$ & 211492 & $s a$ \\
\hline 76 & $514.42 \pm .10$ & 194394 & $d a$ & 138 & $* 472.36 \pm .05$ & 211703 & $s a$ \\
\hline 77 & $513.86 \pm .10$ & 194606 & $d a$ & 139 & $* 472.01 \pm .05$ & 211860 & $s a$ \\
\hline 78 & $513.27 \pm .10$ & 194829 & $d a$ & 140 & $* 471.67 \pm .05$ & 212013 & $s a$ \\
\hline 79 & $512.32 \pm .05$ & 195191 & $s a$ & 141 & $* 470.46 \pm .05$ & 212558 & $s a$ \\
\hline 80 & $510.46 \pm .05$ & 195902 & sa & 142 & $* 469.40 \pm .05$ & 2130 & $s a$ \\
\hline 81 & $509.54 \pm .03$ & 196255 & $s a$ & 143 & $* 468.67 \pm .05$ & 213370 & $s a$ \\
\hline 82 & $509.27 \pm .03$ & 196359 & sa & 144 & $* 468.15 \pm .05$ & 213607 & $s a$ \\
\hline 83 & ${ }^{d} 507.85 \pm .10$ & 196909 & $d w$ & 145 & $467.72 \pm .05$ & 213803 & $s a$ \\
\hline 84 & $506.69 \pm .05$ & 197359 & $s w$ & $\overline{1} \overline{4} \overline{6}$ & $467.54 \pm .05$ & 213885 & sa \\
\hline 85 & $506.36 \pm .05$ & 197488 & $+s w$ & 147 & $467.02 \pm .05$ & 214124 & $d a$ \\
\hline 86 & $505.89 \pm .10$ & 197671 & $s a$ & 148 & $466.30 \pm .2$ & 214450 & $d a$ \\
\hline 87 & $505.42 \pm .03$ & 197855 & $-s x$ & 149 & $465.80 \pm .05$ & 214684 & $s a$ \\
\hline 88 & $504.40 \pm .05$ & 198255 & $-s x$ & 150 & $465.10 \pm .05$ & 215008 & $d a$ \\
\hline 89 & $503.64 \pm .05$ & 198555 & $-\mid s x$ & 151 & $464.35 \pm .05$ & 215355 & $s a$ \\
\hline 90 & $502.87 \pm .05$ & 198859 & $-s x$ & 152 & $463.27 \pm .07$ & 215857 & $s a$ \\
\hline 91 & $502.13 \pm .03$ & 199152 & $-s w$ & 153 & $462.63 \pm .07$ & 216155 & $s a$ \\
\hline 92 & $501.74 \pm .05$ & 199306 & $s w$ & 154 & $462.23 \pm .07$ & 216343 & $s a$ \\
\hline 93 & $501.39 \pm .05$ & 199446 & $s w$ & 155 & $* 461.00 \pm .05$ & 216920 & $+s a$ \\
\hline 94 & $500.37 \pm .05$ & 199852 & $-s w$ & 156 & $460.31 \pm .10$ & 217245 & $d d a$ \\
\hline
\end{tabular}


TABLE 2. Code number, wavelength $(\lambda)$, wave number $(\nu)$, and profile type for all observed Xe resonances, listed in order of decreasing wavelength-Continued

\begin{tabular}{|c|c|c|c|c|c|c|c|}
\hline Number & Wavelength $(\AA)$ & $\begin{array}{l}\text { Wave number } \\
\quad\left(\mathrm{cm}^{-1}\right)\end{array}$ & Comment $^{\mathrm{a}}$ & Number & W avelength $(\AA)$ & $\begin{array}{l}\text { Wave number } \\
\left(\mathrm{cm}^{-1}\right)\end{array}$ & Comment $^{\mathrm{a}}$ \\
\hline 157 & $* 459.68 \pm .05$ & 217543 & $s a$ & 206 & $* 432.31 \pm .05$ & 231315 & $d a$ \\
\hline 158 & $459.23 \pm .05$ & 217756 & $d a$ & 207 & $431.87 \pm .05$ & 231551 & $s a$ \\
\hline 159 & $* 458.63 \pm .05$ & 218041 & $s a$ & 208 & $* 431.55 \pm .05$ & 231723 & $s a$ \\
\hline 160 & $* 458.04 \pm .05$ & 218322 & $s a$ & 209 & $* 431.26 \pm .05$ & 231879 & $s a$ \\
\hline 161 & $456.80 \pm .2$ & 218910 & $d a$ & 210 & $* 430.90 \pm .05$ & 232072 & $s a$ \\
\hline 162 & $456.71 \pm .07$ & 218957 & $-s a$ & 211 & $* 430.63 \pm .05$ & 232218 & $s a$ \\
\hline 163 & $456.42 \pm .05$ & 219096 & $-s a$ & 212 & $* 430.45 \pm .05$ & 232315 & $s a$ \\
\hline 164 & $455.83 \pm .05$ & 219380 & $s a$ & 213 & $* 430.31 \pm .05$ & 232391 & $s a$ \\
\hline 165 & $455.40 \pm .05$ & 219587 & $s a$ & 214 & $429.67 \pm .05$ & 232737 & $+s a$ \\
\hline 166 & $454.95 \pm .05$ & 219804 & $-s a$ & 215 & $428.36 \pm .05$ & 233448 & $s a$ \\
\hline 167 & $* 454.34 \pm .05$ & 220099 & $-s a$ & 216 & $428.14 \pm .05$ & 233568 & $s a$ \\
\hline 168 & $454.00 \pm .05$ & 220264 & $s a$ & 217 & $427.92 \pm .05$ & 233689 & $s a$ \\
\hline 169 & $453.70 \pm .05$ & 220410 & $s a$ & 218 & $426.61 \pm .05$ & 234406 & $+s a$ \\
\hline 170 & $* 453.42 \pm .05$ & 220546 & $-s a$ & 219 & $425.88 \pm .05$ & 234808 & $d a$ \\
\hline 171 & $453.11 \pm .07$ & 220697 & $s a$ & 220 & $425.51 \pm .05$ & 235012 & $d a$ \\
\hline 172 & $452.70 \pm .07$ & 220897 & $-s x$ & 221 & $424.97 \pm .05$ & 235311 & $s a$ \\
\hline 173 & $* 452.35 \pm .10$ & 221068 & $d d a$ & 222 & $424.47 \pm .10$ & 235588 & $d a$ \\
\hline 174 & $452.06 \pm .07$ & 221210 & $s a$ & 223 & $424.02 \pm .10$ & 235838 & $d a$ \\
\hline 175 & $450.33 \pm .05$ & 222059 & $-d a$ & 224 & $423.43 \pm .10$ & 236167 & $d a$ \\
\hline 176 & $449.67 \pm .05$ & 222385 & $d a$ & 225 & $422.73 \pm .10$ & 236558 & $d a$ \\
\hline 177 & $449.15 \pm .05$ & 222643 & $d a$ & 226 & $420.40 \pm .2$ & 237870 & $d d a$ \\
\hline 178 & $448.42 \pm .05$ & 223005 & $d a$ & 227 & $418.00 \pm .2$ & 239230 & $d d a$ \\
\hline 179 & $448.01 \pm .05$ & 223209 & $s a$ & 228 & $413.70 \pm .10$ & 241721 & $d a$ \\
\hline 180 & $447.61 \pm .05$ & 223409 & $d a$ & 229 & $410.30 \pm .10$ & 243724 & $d a$ \\
\hline 181 & $445.91 \pm .05$ & 224260 & $s a$ & 230 & $408.94 \pm .10$ & 244535 & $s a$ \\
\hline 182 & $* 445.61 \pm .05$ & 224411 & $d a$ & 231 & $408.50 \pm .10$ & 244798 & $s a$ \\
\hline 183 & $445.41 \pm .05$ & 224512 & $d a$ & 232 & $407.67 \pm .10$ & 245296 & $s a$ \\
\hline 184 & $444.93 \pm .05$ & 224754 & $s a$ & 233 & $406.32 \pm .10$ & 246111 & $s a$ \\
\hline 185 & $444.38 \pm .05$ & 225033 & $s a$ & 234 & $406.07 \pm .10$ & 246263 & $s a$ \\
\hline 186 & $* 443.73 \pm .07$ & 225362 & $d a$ & 235 & $404.96 \pm .10$ & 246938 & $s a$ \\
\hline 187 & $443.27 \pm .05$ & 225596 & $d a$ & 236 & $404.63 \pm .10$ & 247139 & $s a$ \\
\hline 188 & $442.91 \pm .05$ & 225779 & $d a$ & 237 & $403.87 \pm .10$ & 247604 & $s a$ \\
\hline 189 & $* 442.41 \pm .07$ & 226035 & $d a$ & 238 & $401.90 \pm .2$ & 248820 & $d a$ \\
\hline 190 & $* 441.46 \pm .10$ & 226521 & $s a$ & 239 & $401.24 \pm .10$ & 249227 & $s a$ \\
\hline 191 & $* 441.01 \pm .07$ & 226752 & $s a$ & 240 & $400.90 \pm .10$ & 249439 & sa \\
\hline 192 & $* 440.59 \pm .07$ & 226968 & $s a$ & 241 & $399.94 \pm .10$ & 250038 & $d a$ \\
\hline 193 & $* 440.33 \pm .07$ & 227102 & $s a$ & 242 & $399.56 \pm .10$ & 250275 & $d a$ \\
\hline 194 & $439.92 \pm .07$ & 227314 & $d a$ & 243 & $399.00 \pm .2$ & 250630 & $d a$ \\
\hline 195 & $439.30 \pm .07$ & 227635 & $d a$ & 244 & $397.72 \pm .15$ & 251433 & $d a$ \\
\hline 196 & $438.29 \pm .07$ & 228159 & $s a$ & 245 & $396.70 \pm .2$ & 252080 & $d a$ \\
\hline 197 & $437.64 \pm .07$ & 228498 & $s a$ & 246 & $395.66 \pm .15$ & 252742 & $d a$ \\
\hline 198 & $437.22 \pm .05$ & 228718 & $s a$ & 247 & $392.28 \pm .15$ & 254920 & $d d a$ \\
\hline 199 & $436.82 \pm .05$ & 228927 & $s a$ & 248 & $387.39 \pm .15$ & 258138 & $d a$ \\
\hline 200 & $436.60 \pm .05$ & 229043 & $s a$ & 249 & $385.62 \pm .15$ & 259323 & $d a$ \\
\hline 201 & $436.15 \pm .05$ & 229279 & $+d a$ & 250 & $384.56 \pm .15$ & 260037 & $d a$ \\
\hline 202 & $435.75 \pm .05$ & 229489 & $d a$ & 251 & $383.33 \pm .15$ & 260872 & $d a$ \\
\hline 203 & $435.16 \pm .05$ & 229801 & $s a$ & 252 & $377.20 \pm .15$ & 265111 & $d a$ \\
\hline 204 & $* 434.34 \pm .05$ & 230234 & $d a$ & 253 & $375.34 \pm .15$ & 266425 & $d a$ \\
\hline 205 & $* 433.30 \pm .05$ & 230787 & $d a$ & & & & \\
\hline
\end{tabular}

a Sign plus (+) or minus (-) represents the sign of $q$ in the Fano [12] representation of noninteracting resonances. The letters $s, d, d d$, give a very rough idea of the sharpness or diffuseness of lines on a photographic plate. Naturally, this apparent width varies with gas pressure. The letters $x w$, or $a$ : indicate specifically if the resonance has been measured at $(x)$ the point of highest rate of change of plate density. ( $w$ ) the point of maximum plate density, or $(a)$ the point of minimum plate density (maximum absorption); e.g., $s w$, means a sharp resonance of low unknown $(q)$ measured at the peak transmission point.

${ }^{b}$ Errors shown represent the estimated absolute uncertainty. In the case of Xe the relative accuracies are of the order of $0.02 \AA$ better than the estimated absolute uncertainty.

${ }^{\text {c }}$ Resonances denoted by an asterisk $\left(^{*}\right)$ are listed in table 4 as possible members of Rydberg series.

d Possible double resonance. 
The absorption cross section in the region of a resonance can be parameterized in the form:

$$
\sigma(E)=\sigma_{a} \frac{(q+\epsilon)^{2}}{1+\epsilon^{2}}+\sigma_{b}
$$

with $\epsilon=\left(E-E_{r}\right) / \frac{1}{2} \Gamma$, where the quantities $E$ and $E_{r}$ are the photon energy and the resonance energy $(\epsilon=0)$, respectively. The parameter $q$ is the resonance profile index and is defined in terms of transition matrix elements between the ground state, the modified discrete state and the continuum states. The halfwidth of the resonance is $\Gamma$, the quantity $\sigma_{a}$ is the cross section associated with the fraction of the available continua with which the discrete state interacts, and $\sigma_{b}$ is the cross section associated with the fraction of the continua which does not enter into the interaction. The parameter $\rho$, called the correlation index [12], is obtained from the relation $\rho^{2}=\frac{\sigma_{a}}{\sigma_{a}+\sigma_{b}}$.

Experimentally, when $q$ is positive, a region of low absorption at low energy immediately precedes a region of increased absorption at higher energy. For a negative $q$, the reverse is true. If $q$ is of the order of 1 , the resonance appears quite asymmetric. If $q$ is large and $\rho^{2}$ is small, the resonance looks like a conventional Lorentz absorption profile (absorption-type resonance), but if $q$ is close to zero, the resonance has only a reduced-absorption zone (window-type resonance). When possible, in tables 1 and 2 the sign $q$ is given. Where the letter $x, a$, and $w$ are used, the resonance appears to be an asymmetric, absorption or window-type resonance, respectively, and has been measured as such.

\subsection{One-Electron Transitions}

The most prominent series should be those associated with one-electron transitions. This was found to be the case in $\mathrm{Ne}$ and $\mathrm{Ar}$, and is equally true in $\mathrm{Kr}$ and $\mathrm{Xe}$. The ground state of the noble gases is a ${ }^{1} \mathrm{~S}_{0}$. In a photoexcitation process, therefore, the upper state involved must have $J=1$, regardless of the coupling conditions.

Since strict $L-S$ coupling is not applicable, the strongest Rydberg series would be due to transitions:

$$
\begin{aligned}
& \mathrm{Kr}: 4 s^{2} 4 p^{6}{ }^{1} \mathrm{~S}_{0} \rightarrow 4 s 4 p^{6}\left({ }^{2} \mathrm{~S}_{1 / 2}\right) n p ; n=5,6 \text {. . (two } J=1 \\
& \text { components) } \\
& \text { Xe: } 5 s^{2} 5 p^{6}{ }^{1} \mathrm{~S}_{0} \rightarrow 5 s 5 p^{6}\left({ }^{2} \mathrm{~S}_{1 / 2}\right) n p ; n=6,7 \text {. . (two } J=1 \\
& \text { components) }
\end{aligned}
$$

The upper configurations have only two $J=1$ states, and transitions to upper states of other $J$ cannot occur due to the rigorous selection rules: $\Delta J=0, \pm 1$; $0 \nrightarrow 0$.

Thus we might expect to resolve at low $n$ values, the two $J=1$ members converging to the known ${ }^{2} \mathrm{~S}_{1 / 2}$ limits of $\mathrm{Kr}$ II or Xe II. (This limit occurs in $\mathrm{Kr}$ at $450.62 \AA$ and in $\mathrm{Xe}$ at $529.91 \AA$ ).
In the case of $\mathrm{Kr}$, we see three "strong" window resonances in the region where the first members of the one-electron excitation series should occur. In $\mathrm{Xe}$, one "strong" resonance and three somewhat weaker resonances are observed. "Strong" in this case means high visibility rather than a large oscillator strength, since the visibility of resonances depends also on the interference effects. Additionally, the ability to see window resonances is photographically enhanced at lower pressures.

The choice of the two $J=1$ components of the first member of the Rydberg series is by no means unambiguous. The two components chosen in $\mathrm{Kr}$ are seen in the top photograph of figure 1 and again in the densitometer trace, figure 2 . The resonances are denoted by their code numbers 3 and 5 , see table 1 . The wavelengths, principal quantum numbers $(n)$ and effective quantum numbers $\left(n^{*}\right)$ are given in table 3 , part A. Recent work appears to support the above choice. Reader et al. [13] noted that the effective quantum number of the $5 p$ electron in the $4 s 4 p^{6} 5 p$ state in the isoelectronic sequence $\mathrm{ZrV}, \mathrm{Nb} \mathrm{VI}$, and Mo VII consistently differed from the $n^{*}$ in the $4 s^{2} 4 p^{6} 5 p$ state of $\mathrm{Nb} \mathrm{V}$, Mo VI, and Te VII by 0.040 . Extrapolating this relationship the $n^{*}$ for the $4 s 4 p^{6} 5 p$ state of $\mathrm{Kr}$ I can be obtained from the $n^{*}$ for the $4 s^{2} 4 p^{6} 5 p$ state of $\mathrm{Rb}_{\mathrm{I}}(2.289)$. The result, 2.329 , is in good agreement with the value of 2.323 for the assigned resonance to this state in table 3 .

In $\mathrm{Xe}$, the most prominent member of the quartet of resonances (Code Nos. 1, 2, 3, 4) has been chosen as the first member of the one-electron excitation series: $3,16,28$ etc. This series can be seen in the lower photograph of figure 1 and also in the densitometer trace figure 3 . The wavelengths and effective quantum numbers are given in table 4, part A. A choice has not been made for the second $J=1$ component in Xe, although it is likely to be resonance 1 or 2 .

Recent work [13] suggests that the main $J=1$ component in Xe should have an effective quantum number $n^{*}=2.391$. If resoance 4 is taken as the first member of the series, its effective quantum number is 2.398 . Resonance 3 chosen here gives $n^{*}=2.359$. This choice was made upon the basis of the great similarity in resonance profiles of line 3 and the following members of the series, resonances 16 and 28 . Figure 3 clearly shows that line 3 is asymmetric, with a low positive $q$, whereas resonance 4 has a $q$ of the opposite sign. In fact, Ederer [10] has shown that the values of $q$ and $\rho^{2}$ for resonance 3 are $0.23( \pm .04)$ and $0.65( \pm .03)$, for resonance 4 are $-0.14( \pm .04)$ and $0.50( \pm .04)$ and for resonance 16 are $0.16( \pm .04)$ and $0.67( \pm .02)$. In addition, we can compare the equivalent one-electron excitation states in $\mathrm{Ne}, \mathrm{Ar}, \mathrm{Kr}$, and $\mathrm{Xe}$ and the way in which the first member of the Rydberg series is always depressed (moved to longer wavelengths) relative to a simple theoretical estimate of its location, due to screening effects. The increase in this screening effect from $\mathrm{Ne}$ through $\mathrm{Kr}$ leads us to expect the strongest $J=1$ component in Xe to lie almost exactly where it is. Nevertheless, it is conceivable that configuration interaction may cause resonances to interchange intensi- 


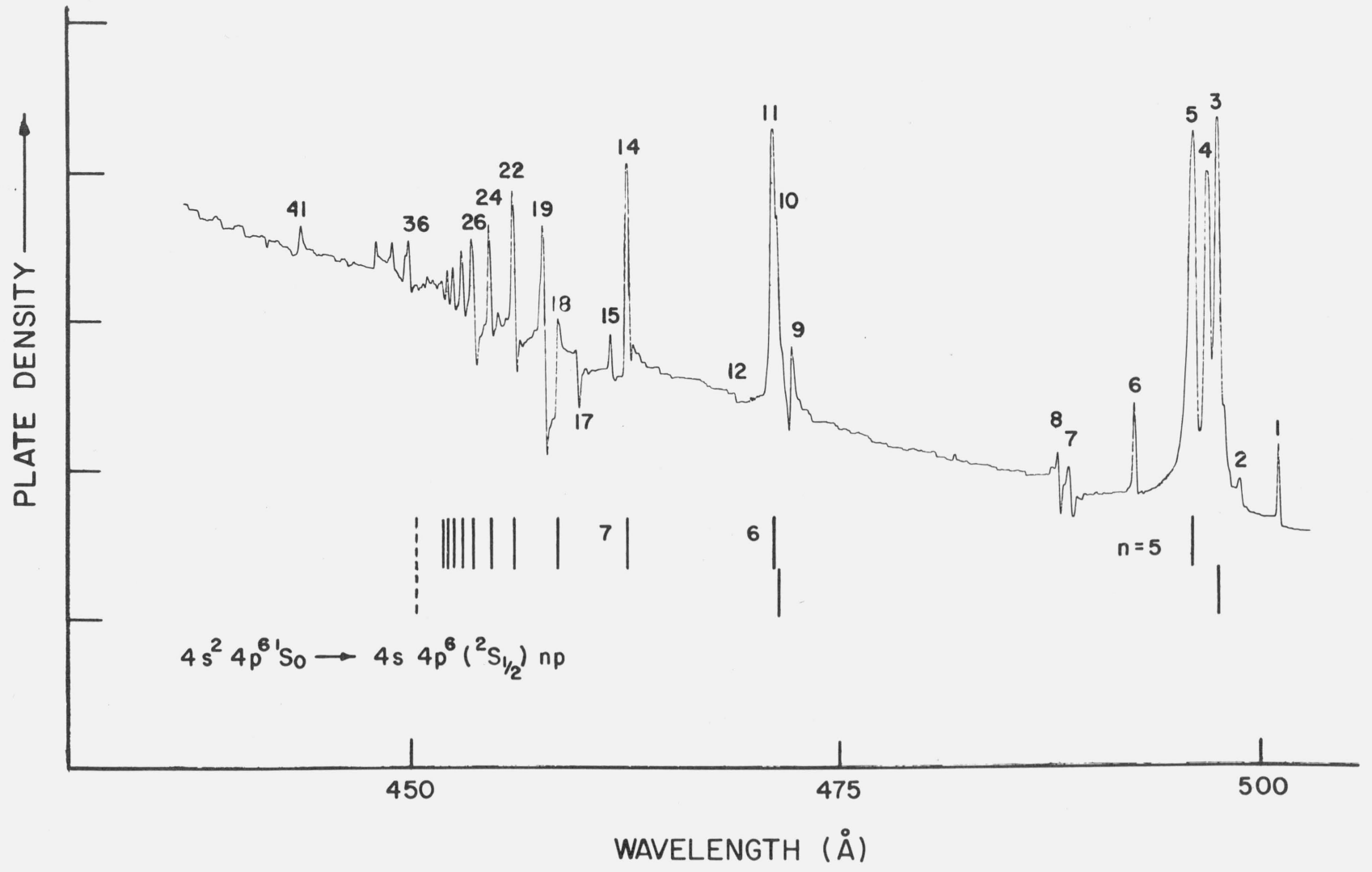

FIGURE 2. A microdensitometer trace of the absorption spectrum of $\mathrm{Kr}$ in the region $435-500 \AA$.

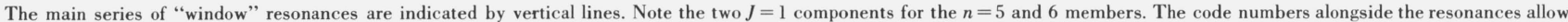
determination of their wavelengths (and in some cases classification) via tables 1 and 3 .

TABLE 3. Resonances in Kr I grouped as members of possible series converging to the limits indicated

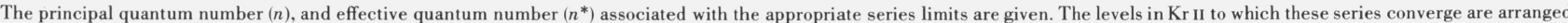
in order of increasing energy. Their positions are obtained from data [18] on the Kr II, added to $112915 \mathrm{~cm}^{-1}$, the first ionization energy of $\mathrm{Kr}$.

\begin{tabular}{|c|c|c|c|c|c|c|c|}
\hline Code number & $\lambda(\AA)$ & $n$ & $n^{*}$ & Code number & $\lambda(\AA)$ & $n$ & $n^{*}$ \\
\hline \multicolumn{4}{|c|}{ (A) Limit: $-4 s 4 p^{6}\left({ }^{2} \mathrm{~S}_{1 / 2}\right)=221915 \mathrm{~cm}^{-1}$; Series: $-4 s 4 p^{6} n p$} & \multicolumn{4}{|c|}{ (B) Unknown limit ${ }^{\mathrm{a}}=240300 \mathrm{~cm}^{-1}$} \\
\hline 5 & 496.07 & 5 & 2.323 & 63 & 417.96 & 13 & a $(9.35)$ \\
\hline 11 & 471.23 & 6 & 3.363 & 64 & 417.62 & 14 & (10.18) \\
\hline 14 & 462.71 & 7 & 4.351 & 65 & 417.39 & 15 & (10.88) \\
\hline 18 & 458.69 & 8 & 5.303 & 66 & 417.22 & 16 & (11.49) \\
\hline 22 & 456.12 & 9 & 6.406 & \multirow{2}{*}{\multicolumn{4}{|c|}{$(\mathrm{C}):-4 p^{4}\left({ }^{1} \mathrm{~S}\right) 5 s\left({ }^{2} \mathrm{~S}_{1 / 2}\right)=258727 \mathrm{~cm}^{-1} ;$ Series: $-4 p^{4} 5 s n p$}} \\
\hline 24 & 454.73 & 10 & 7.399 & & & & \\
\hline 26 & 453.73 & 11 & 8.498 & & & & \\
\hline 27 & 453.15 & 12 & 9.42 & 86 & 391.44 & 8 & 5.59 \\
\hline 28 & 452.68 & 13 & 10.43 & 87 & 390.49 & 9 & 6.45 \\
\hline 29 & 452.34 & 14 & 11.41 & 89 & 389.48 & 10 & 7.46 \\
\hline 30 & 452.07 & 15 & 12.43 & 90 & 388.84 & 11 & 8.41 \\
\hline 32 & 451.86 & 16 & 13.44 & 91 & 388.37 & 12 & 9.41 \\
\hline 33 & 451.69 & 17 & 14.47 & 92 & 388.05 & 13 & 10.33 \\
\hline 34 & 451.56 & 18 & 15.44 & 93 & 387.79 & 14 & 11.33 \\
\hline 35 & 451.45 & 19 & 16.43 & 94 & 387.60 & 15 & 12.27 \\
\hline & & & & 96 & 387.08 & 16 & 13.21 \\
\hline \multicolumn{4}{|c|}{ Second series of two available, having $J=1$ final state } & \multicolumn{4}{|c|}{ (D) Limit: $-4 p^{4}\left({ }^{1} \mathrm{D}\right) 4 d\left({ }^{2} \mathrm{D}_{5 / 2}\right)=262429 \mathrm{~cm}^{-1} ;$ Series: $-4 p^{4} 4 d n p$} \\
\hline 3 & 497.50 & 5 & 2.304 & 99 & 385.03 & 9 & 6.37 \\
\hline 10 & 471.48 & 6 & 3.343 & 101 & 384.03 & 10 & 7.35 \\
\hline
\end{tabular}


TABLE 3. Resonances in $\mathrm{Kr} \mathrm{I}$ grouped as members of possible series converging to the limits indicated-Continued

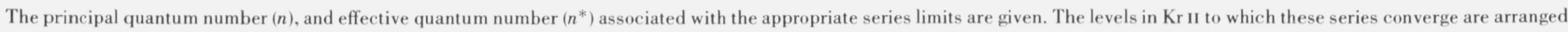
in order of increasing energy. Their positions are obtained from data [18] on the $\mathrm{Kr} \mathrm{II}$, added to $112915 \mathrm{~cm}^{-1}$, the first ionization energy of $\mathrm{Kr} \mathrm{I}$.

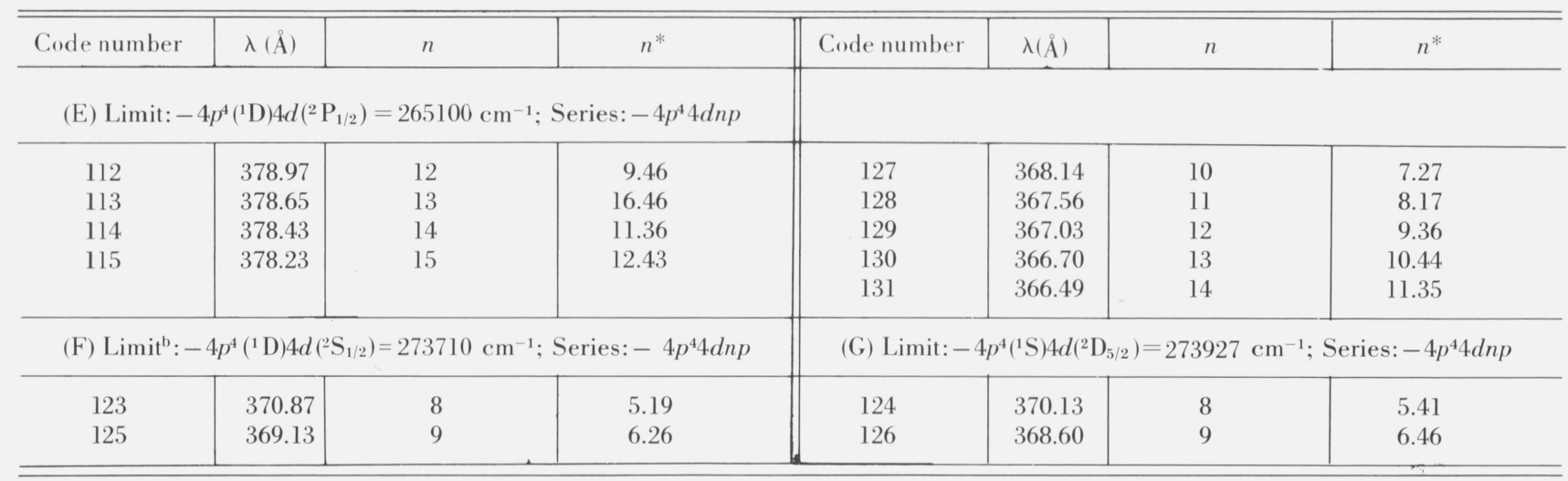

a The nearest known limit $[18]$ is $\left({ }^{1} \mathrm{D}\right) 5 s\left({ }^{2} \mathrm{D}_{3 / 2}\right)$ at $240512 \mathrm{~cm}^{-1}$. One could assume this limit to be the apppropriate one, giving the values of $n^{*}$ shown in parentheses. Noninteger difference in $n^{*}$ through the series could possibly be explained by configuration interaction effects.

b This limit was previously assigned [19] to $4 p^{4}\left({ }^{3} \mathrm{P}\right) 5 d\left({ }^{4} \mathrm{P}_{1 / 2}\right)$. It was recently reassigned [18] by Minnhagen.

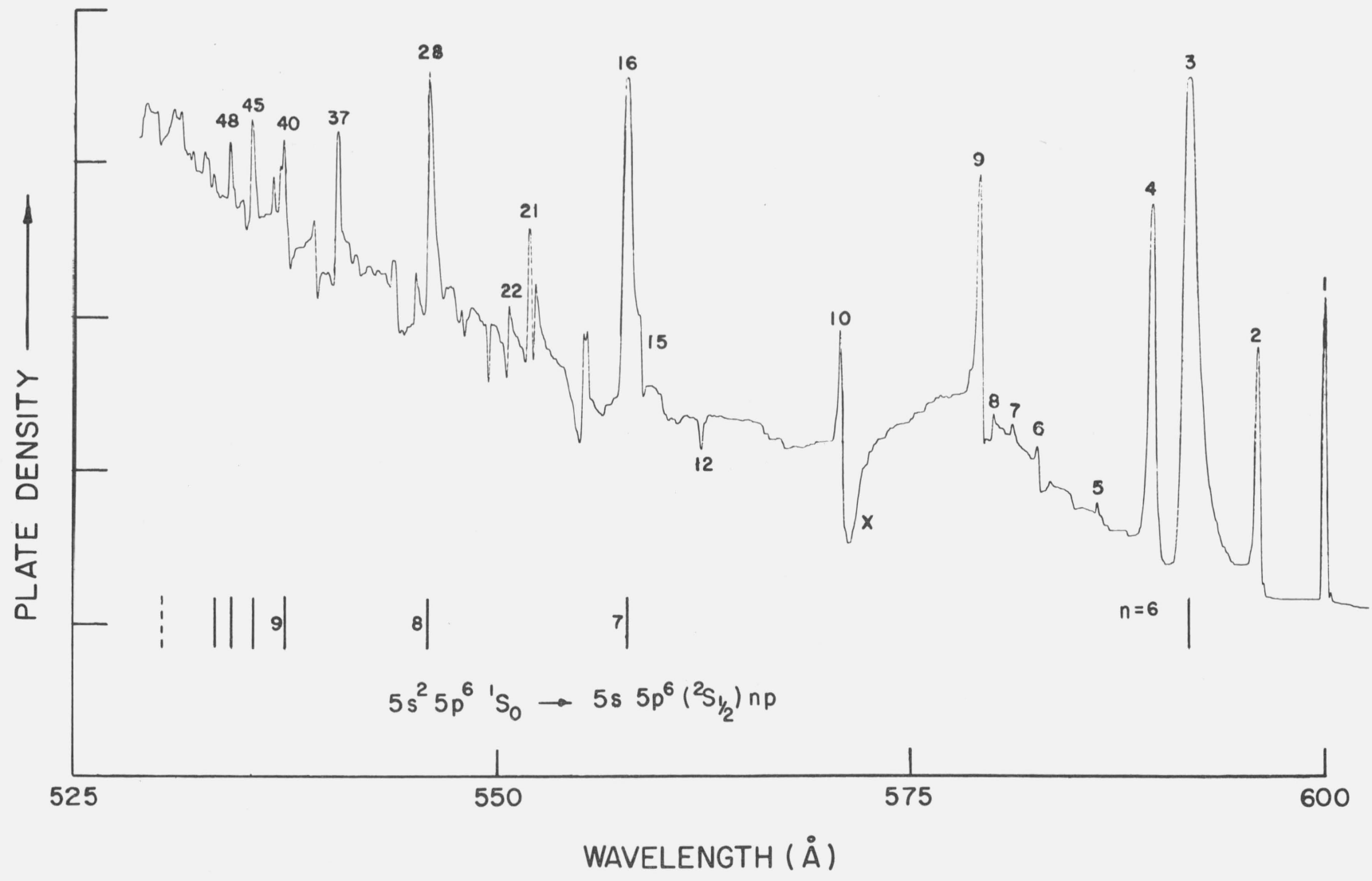

FigURE 3. A microdensitometer trace of the absorption spectrum of $\mathrm{Xe}$ in the region $525-600 \AA$.

The main series of "window" resonances is denoted by vertical lines. The code numbers allow determination of wavelengths (and in some cases classification) via tables 2 and 4 . The absorption feature labeled X, is the third order of an absorption line [4] at 190.41 $\AA$. 


\begin{tabular}{|c|c|c|c|c|c|c|c|}
\hline Code number & $\lambda(\AA)$ & $n$ & $n^{*}$ & Code number & $\lambda(\AA)$ & $n$ & $n^{*}$ \\
\hline \multicolumn{4}{|c|}{ (A) Limit: $-5 s 5 p^{6}\left({ }^{2} \mathrm{~S}_{1 / 2}\right)=188708 \mathrm{~cm}^{-1} ;$ Series: $-5 s 5 p^{6} n p$} & \multicolumn{4}{|c|}{ (E) Limit: $-5 p^{4}\left({ }^{3} \mathrm{P}\right) 6 p\left({ }^{4} \mathrm{D}_{3 / 2}\right)=214617 \mathrm{~cm}^{-1}$; Series: $-5 p^{4} 6 p n d$} \\
\hline \multirow{11}{*}{$\begin{array}{r}3 \\
16 \\
28 \\
a \\
37 \\
40 \\
45 \\
48 \\
b 49 \\
51 \\
55 \\
57 \\
58 \\
59\end{array}$} & \multirow{11}{*}{$\begin{array}{l}591.77 \\
557.83 \\
546.08 \\
540.54 \\
537.46 \\
535.55 \\
534.27 \\
533.34 \\
532.76 \\
531.21 \\
530.93 \\
530.83 \\
530.74\end{array}$} & \multirow{11}{*}{$\begin{array}{r}6 \\
7 \\
8 \\
9 \\
10 \\
11 \\
12 \\
13 \\
14 \\
19 \\
21 \\
22 \\
23\end{array}$} & \multirow{11}{*}{$\begin{array}{c}2.359 \\
3.409 \\
4.433 \\
5.440 \\
6.438 \\
7.437 \\
8.450 \\
9.52 \\
10.44 \\
15.47 \\
17.48 \\
18.41 \\
19.39\end{array}$} & 141 & 470.46 & 10 & 7.30 \\
\hline & & & & 142 & 469.40 & 11 & 8.34 \\
\hline & & & & 143 & 468.67 & 12 & 9.38 \\
\hline & & & & 144 & 468.15 & 13 & 10.42 \\
\hline & & & & \multirow{2}{*}{\multicolumn{4}{|c|}{ (F) Limit: $-5 p^{4}\left({ }^{3} \mathrm{P}\right) 6 p\left({ }^{4} \mathrm{~S}_{3 / 2}\right)=219463 \mathrm{~cm}^{-1}$; Series: $-5 p^{4} 6 p n d$}} \\
\hline & & & & & & & \\
\hline & & & & \multirow{4}{*}{$\begin{array}{l}155 \\
157 \\
159 \\
160\end{array}$} & 461.00 & 9 & 6.57 \\
\hline & & & & & 459.68 & 10 & 7.56 \\
\hline & & & & & 458.63 & 11 & 8.78 \\
\hline & & & & & 458.04 & 12 & 9.80 \\
\hline & & & & \multicolumn{4}{|c|}{ (G) Limit: $-5 p^{4}\left({ }^{1} \mathrm{~S}\right) 5 d\left({ }^{2} \mathrm{D}_{3 / 2}\right)=222136 \mathrm{~cm}^{-1} ;$ Series: $-5 p^{4} 5 d n p$} \\
\hline \multicolumn{4}{|c|}{ (B) Limit: $-5 p^{4}\left({ }^{3} \mathrm{P}\right) 6 s\left({ }^{4} \mathrm{P}_{5 / 2}\right)=190902 \mathrm{~cm}^{-1}$; Series: $-5 p^{4} 6 s n p$} & \multirow{2}{*}{$\begin{array}{l}167 \\
170 \\
173\end{array}$} & \multirow{2}{*}{$\begin{array}{l}454.34 \\
453.42 \\
452.35\end{array}$} & \multirow{3}{*}{$\begin{array}{l}11 \\
12 \\
13\end{array}$} & \multirow{2}{*}{$\begin{array}{l}7.34 \\
8.31 \\
9.41\end{array}$} \\
\hline \multirow{6}{*}{$\begin{array}{l}22 \\
38 \\
47 \\
54 \\
62 \\
65\end{array}$} & \multirow{6}{*}{$\begin{array}{l}550.76 \\
539.33 \\
534.58 \\
531.37 \\
529.34 \\
528.05\end{array}$} & \multirow{6}{*}{$\begin{array}{r}7 \\
8 \\
9 \\
10 \\
11 \\
12\end{array}$} & \multirow{6}{*}{$\begin{array}{l}3.429 \\
4.472 \\
5.346 \\
6.364 \\
7.431 \\
8.480\end{array}$} & & & & \\
\hline & & & & \multirow{2}{*}{\multicolumn{4}{|c|}{ (H) Limit: $-5 p^{4}\left({ }^{1} \mathrm{D}\right) 6 p\left({ }^{2} \mathrm{~F}_{7 / 2}\right)=227898 \mathrm{~cm}^{-1}$; Series: $-5 p^{4} 6 p n d$}} \\
\hline & & & & & & & \\
\hline & & & & \multirow{7}{*}{$\begin{array}{l}182 \\
186 \\
189 \\
190 \\
191 \\
192 \\
193\end{array}$} & & & \\
\hline & & & & & 445.61 & 8 & 5.61 \\
\hline & & & & & 443.73 & 9 & 6.58 \\
\hline (C) Limit:- & P) $6 s\left({ }^{4} \mathrm{P}_{3}\right.$ & & $5 p^{4} 6 s n p$ & & $\begin{array}{l}442.41 \\
441.46\end{array}$ & $\begin{array}{l}10 \\
11\end{array}$ & $\begin{array}{l}7.67 \\
8.93\end{array}$ \\
\hline \multirow{4}{*}{$\begin{array}{r}31 \\
b^{b} 49 \\
64 \\
69\end{array}$} & & & & & 441.01 & 12 & 9.79 \\
\hline & \multirow{3}{*}{$\begin{array}{l}544.18 \\
533.34 \\
528.42 \\
525.77\end{array}$} & 7 & \multirow{3}{*}{$\begin{array}{l}3.466 \\
4.508 \\
5.480 \\
6.374\end{array}$} & & 440.59 & 13 & 10.87 \\
\hline & & 8 & & & 440.33 & 14 & 11.74 \\
\hline & & $\begin{array}{r}9 \\
10\end{array}$ & & \multicolumn{4}{|c|}{ (I) Limit: $-5 p^{4}\left({ }^{3} \mathrm{P}\right) 7 s\left({ }^{4} \mathrm{P}_{1 / 2}\right)=232895 \mathrm{~cm}^{-1} ;$ Series: $-5 p^{4} 7 s n p$} \\
\hline \multirow{2}{*}{\multicolumn{4}{|c|}{ (D) Unknown Limit ${ }^{c}=212665 \mathrm{~cm}^{-1}$}} & 204 & 434.34 & 10 & 6.42 \\
\hline & & & & 205 & 433.30 & 11 & 7.22 \\
\hline 135 & 474.36 & 8 & 7.691 & $\begin{array}{l}206 \\
208\end{array}$ & $\begin{array}{l}432.31 \\
431.55\end{array}$ & $\begin{array}{l}12 \\
13\end{array}$ & $\begin{array}{l}8.34 \\
9.68\end{array}$ \\
\hline 136 & 473.42 & 9 & 8.742 & 209 & 431.26 & 14 & 10.39 \\
\hline 137 & 472.83 & 10 & 9.68 & 210 & 430.90 & 15 & 11.55 \\
\hline 138 & 472.36 & 11 & 10.68 & 211 & 430.63 & 16 & 12.73 \\
\hline 139 & 472.01 & 12 & 11.68 & 212 & 430.45 & 17 & 13.76 \\
\hline 140 & 471.67 & 13 & 12.97 & 213 & 430.31 & 18 & 14.75 \\
\hline
\end{tabular}

${ }^{a}$ The resonance at $540.62 \AA$ is an alternative choice for the $n=9$ member.

${ }^{b}$ This resonance appears to be double and is used in series (A) and (C).

${ }^{c}$ The nearest $\mathrm{Xe}$ II levels are $5 p^{4}\left({ }^{1} \mathrm{D}\right) 5 d\left({ }^{2} \mathrm{~F}_{5 / 2}, 7 / 2\right)$ at $212585 \mathrm{~cm}^{-1}$ and $212748 \mathrm{~cm}^{-1}$, either of which would allow an $n f$ series.

${ }^{d}$ The quantum defects for this series are more appropriate to a running $p$ electron. However, assuming a correct assignment for the level in Xe II, parity considerations require a running $d$ electron (or an $s$ electron).

ties, and affect their shapes. Good theoretical calculations will be required to resolve this situation.

If we move to higher series members in $\mathrm{Kr}$, we find that resonance 18 has an unusually low effective quantum number and a low intensity (see fig. 2). On the basis of the quantum defect alone, resonance 18 , the $n=8$ member of this series, was chosen as a member of the Rydberg series. Clearly, resonance 19 is much "stronger" (see also Ederer [10]) and was observed by Samson [15] and classified as a member of the series with $n^{*}=5.593$. Once again, there is ambiguity. The remainder of the series is quite definitely established on a basis of quantum defects, intensities and profiles.

In tracing the development of the Xe series, we find the $n=9$ member to be double and an arbitrary choice must be made from resonances 36 and 37 . Other workers [14, 15] did not resolve these resonances. Configuration interactions cause members $n=15$ through 18 and $n=20$ to be missing. 
TABLE 5. A comparison of the wavelengths of some of the resonances measured in the present work and those of other workers

The Siegbahn [16] wavelengths are obtained using the conversion factor $\lambda=12398 / \mathrm{E}(\mathrm{eV})$.

\begin{tabular}{|c|c|c|c|c|c|c|c|c|}
\hline \multicolumn{4}{|c|}{ Krypton } & \multicolumn{5}{|c|}{ Xenon } \\
\hline Code & Present work & Samson [15] & Siegbahn [16] & Code & Present work & Samson [15] & Mansfield [17] & Siegbahn [16] \\
\hline $\begin{array}{r}1 \\
3 \\
4 \\
5 \\
10 \\
14 \\
18 \\
19 \\
22 \\
24 \\
26 \\
27 \\
29 \\
30 \\
32\end{array}$ & $\begin{array}{r}501.23 \\
497.50 \\
496.90 \\
496.07 \\
471.48 \\
462.71 \\
458.69 \\
\text { a } 457.86 \\
456.12 \\
454.73 \\
453.73 \\
453.14 \\
452.32 \\
452.07 \\
451.86\end{array}$ & $\begin{array}{l}501.14 \\
497.44 \\
496.85 \\
496.00 \\
471.55 \\
462.69 \\
457.85 \\
456.10 \\
454.71 \\
453.71 \\
453.14 \\
452.32 \\
452.01 \\
451.85\end{array}$ & $\begin{array}{l}497.1 \\
495.7 \\
471.2 \\
462.8 \\
458.8 \\
\\
456.0 \\
455.0 \\
453.8\end{array}$ & $\begin{array}{r}1 \\
2 \\
3 \\
4 \\
5 \\
6 \\
7 \\
8 \\
9 \\
10 \\
15 \\
16 \\
19 \\
21 \\
22 \\
28 \\
\text { b } 36 \\
37 \\
40 \\
45\end{array}$ & $\left.\begin{array}{l}599.99 \\
595.93 \\
591.77 \\
589.54 \\
586.29 \\
582.74 \\
581.11 \\
579.98 \\
579.16 \\
570.79 \\
558.78 \\
557.83 \\
555.28 \\
552.00 \\
550.76 \\
546.08 \\
540.62 \\
540.54 \\
537.46 \\
535.55\end{array}\right\}$ & $\begin{array}{r}599.95 \\
595.92 \\
591.81 \\
589.62 \\
\\
\\
\\
14579.25 \\
14570.90 \\
557.92 \\
\\
14552.07 \\
\\
546.16 \\
540.71 \\
537.40 \\
535.62\end{array}$ & $\begin{array}{l}599.81 \\
595.83 \\
591.67 \\
589.50 \\
586.24 \\
582.72 \\
581.07 \\
579.94 \\
579.15 \\
570.80 \\
558.78 \\
557.86 \\
555.32 \\
552.02 \\
550.77\end{array}$ & $\begin{array}{l}546.2 \\
540.5\end{array}$ \\
\hline
\end{tabular}

${ }^{a}$ Resonance 18 was chosen in the present work, on the basis of quantum defect; for $4 s 4 p^{6} 8 p$. However, resonance 19 is a considerably stronger line seen also by Samson and assigned to the $8 p$ state.

${ }^{\mathrm{b}}$ Two resonances, equally strong, just resolved.

Finally, in table 5, we see a comparison of the long wavelength resonances in $\mathrm{Kr}$ and $\mathrm{Xe}$ with other workers. In $\mathrm{Kr}$, the present data is compared with the photoabsorption work of Samson [14, 15] and the electron spectroscopic observations of Siegbahn and co-workers [16]. In Xe, comparison is also made with the photoabsorption work of Mansfield [17] (Mansfield measured $\mathrm{Kr}$ also, and was in agreement with the present work for the stronger transitions). The error limits quoted by Samson are $\pm 0.05 \AA$, those of Siegbahn et al. are of the order of $0.2 \AA$. Mansfield gave no estimated error, but he had some calibration problems in the region of $585-600 \AA$ due to lack of standards. The accuracy of the present measurements is also impaired in this region of the spectrum because of slight instrumental errors and this is reflected in the somewhat increased error limits given to the resonances in that region. Almost all of the wavelengths of Samson agree, within the combined error limits, with the present data, even in the range $585-600 \AA$.

The data of Siegbahn et al. was obtained by firing electrons of an energy of several $\mathrm{keV}$ into the noble gases, and measuring the energies (typically 8 to 14 $\mathrm{eV}$ ) of the electrons released in the subsequent autoionization process, with a spherical electrostatic analyzer. Rydberg series of electron lines were found corresponding to transitions from excited states of neutral atoms to the ground state of the ions. By measuring the kinetic energy of electrons produced in the autoionization process:

$$
4 s 4 p^{6} n p \rightarrow 4 s^{2} 4 p^{5}\left({ }^{2} \mathrm{P}_{1 / 2,3 / 2}\right)+e
$$

and knowing the energy of the $4 s^{2} 4 p^{5}\left({ }^{2} P_{1 / 2,3 / 2}\right)$ states of $\mathrm{KrII}$, they were able to calculate the energy of the excited state before autoionization. Since the Siegbahn data was normalized to the present data at certain wavelengths, it cannot be thought of as independent. It is interesting, however, to note that these workers picked out a second $J=1$ component for the $4 s 4 p^{6} 5 p$ state of Kr. Even though the absolute wavelengths of resonances 3 and 5 disagree by $0.3 \AA$, the difference in wavelengths of $1.4 \AA$ is in agreement with the present data. In $\mathrm{Xe}$, however, the implication might be that resonances 3 and 4 are the two $J=1$ components, in agreement with another recent estimate [13], and in disagreement with the present classification. It should be pointed out, however, that excitation by electron bombardment can excite, in general, many transitions not allowed optically and that often such nonoptically allowed transitions are preferred. In addition, the resonance profiles may be completely different.

\subsection{Two-Electron Transitions}

It has been pointed out that only two $J=1$ components can be expected for states such as $4 s 4 p^{6} 5 p$ in $\mathrm{Kr}$. 
The remaining resonances in $\mathrm{Kr}$, such as $1,2,4,6$, 7, 8 etc. (see fig. 2) must therefore be classified, from energy considerations, in terms of two-electron transitions, viz:

$$
\begin{gathered}
\mathrm{Kr}: 4 s^{2} 4 p^{6}{ }^{1} \mathrm{~S}_{0} \rightarrow 4 s^{2} 4 p^{4}\left({ }^{3} \mathrm{P},{ }^{1} \mathrm{D},{ }^{1} \mathrm{~S}\right) n l n^{\prime} l^{\prime} \text { and in } \\
\mathrm{Xe}: 5 s^{2} 5 p^{6}{ }^{1} \mathrm{~S}_{0} \rightarrow 5 s^{2} 5 p^{4}\left({ }^{3} \mathrm{P},{ }^{1} \mathrm{D},{ }^{1} \mathrm{~S}\right) n l n^{\prime} l^{\prime} .
\end{gathered}
$$

Here $l l^{\prime}=s p, s f, p d, p f$.

The term possibilities are just those outlined previously in $\operatorname{Ar}$ [3]. As shown, the grandparent term may be ${ }^{3} \mathrm{P},{ }^{1} \mathrm{D}$, or ${ }^{1} \mathrm{~S}$. Taking the simplest case of $5 s 5 p$ in $\mathrm{Kr}$, the number of possible $J=1$ components is 14 , with 3 of these being ${ }^{1} \mathrm{P}_{1}$, in strict $L-S$ coupling. We know, however, that $L-S$ coupling does not prevail, and we may expect a large number of excited states with $J=1$. Experimentally, one observes few of this potentially large number of resonances.

As in Ar, we can make crude estimates of the expected positions of resonances from the known levels of the ion $[18,19]$ and typical quantum defects for the $s, p, d$, and $f$ electrons. Because the spectra observed are quite rich and because of screening and configuration interaction effects, such estimates cannot be sufficiently accurate to establish definite assignments.

In the cases of $\mathrm{Ne}$ and $\mathrm{Ar}$ a number of low-lying two-electron excitation states were identified. In $\mathrm{Kr}$ and $\mathrm{Xe}$ virtually none have been assigned. In the absence of good calculations, we can only identify series with high-lying members. Intensity sharing interactions appear to be responsible for the fact that we see any series at all.

In both $\mathrm{Kr}$ and $\mathrm{Xe}$, quantum defects have been calculated for all the resonances to the available $[18,19]$ known limits. As a result of this analysis, only 28 resonances in $\mathrm{Kr}$ and 43 in Xe have been grouped into probable Rydberg series. In the Ar case, one experimental fact was very obvious - that of all the possible excited configurations, those of the type:$3 p^{4} 3 d n l$ and $3 p^{4} 4 s n l$ dominated the spectrum. Series such as $3 p^{4} 4 p n s$ were hardly in evidence. This tendency is equally pronounced in $\mathrm{Kr}$, where all of the series are of the type $4 p^{4} 5 s n p$ or $4 p^{4} 4 d n p$.

Turning to table 3 (B), the unidentified series having a limit at $240300 \mathrm{~cm}^{-1}$ may be due to transitions to states $\left({ }^{1} \mathrm{D}\right) 5 s\left({ }^{2} \mathrm{D}_{3 / 2}\right) n p$. However, using Minnhagen's value for this limit [18], we must then invoke certain configuration interaction effects to explain the unusual run of effective quantum numbers (inside the brackets).

In $\mathrm{Xe}$, if the present tentative assignments are correct, the emphasis on running electrons of odd parity is no longer evident. Indeed, there are apparently two series of the type $5 p^{4} 6 p n d$. Another series is tentatively labelled 6pnd (table 4. (E)) but the defects are more appropriate for a running $p$ electron - forbidden on the grounds of nonconservation of parity if the state in Xe II has been correctly identified [19].
Also in table 4, the series (D) with an unidentified limit, may well be associated with a series of the type $5 p^{4} 5 d n f$.

Some of the more obvious series in $\mathrm{Kr}$ and $\mathrm{Xe}$ are indicated by lines above and below the spectra of figure 1 . In $\mathrm{Kr}$ we can see the series A, B, C, F and G of table 3 ; in Xe we see the series A, B, D and I of table 4 .

\section{Conclusions}

The one- and two-electron excitation resonances listed into the series above represent only one quarter of those observed. The basic problem is one of unfolding the complicated configuration interactions present, which displace levels from the locations expected on simple theoretical grounds, and produce intensity anomalies. Even within a single series, the profiles of the resonances may change with increasing principal quantum number. In others, only high series members are evident. It appears that a substantial theoretical effort will be required to analyze the spectra further, even in those regions where relatively few resonances occur. Original prints of the spectra shown in figure 1 can be made available to those interested in furthering the interpretation.

\section{References}

[1] Madden, R. P., and Codling, K., Astrophys. J. 141, 364 (1965).

[2] Codling, K., Madden, R. P., and Ederer, D. L., Phys. Rev. 155, 26 (1967).

[3] Madden, R. P., Ederer, D. L., and Codling, K., Phys. Rev. 177, $136(1969)$

[4] Codling, K., and Madden, R. P., Phys. Rev. Letters 12, 106 (1964).

[5] Codling, K., and Madden, R. P., Appl. Opt. 4, 1431 (1965).

[6] Beutler, H., Zeit. Phys. 93, 177 (1935).

[7] Samson, J. A. R., Phys. Rev. 132, 2122 (1963).

[8] Madden, R. P., and Codling, K., J. Opt. Soc. Am. 54, 268 (1964).

[9] Codling, K., and Madden, R. P., Phys. Rev. A4, 2261, (1971).

[10] Ederer, D. L., Phys. Rev. A4, 2263, (1971).

[11] Madden, R. P., Ederer, D. L., and Codling, K., Appl. Opt. 6, 31 (1967).

[12] Fano, U., and Cooper, J. W., Phys. Rev. 137, Al364 (1965).

[13] Reader, J., Epstein, G. L., and Ekberg, J. O., J. Opt. Soc. Am. (in press).

[14] Samson, J. A. R., Phys. Letters 8, 107 (1964).

[15] Samson, J. A. R., Advances in Atomic and Molecular Physics (Academic Press Inc. New York 1966) Vol. 2, p. 177.

[16] Siegbahn, K., Nordling, C., Johansson, C., Hedman, J., Heden, P. F., Hamrin, K., Gelius, U., Bergmark, T., Werme, L. O., Manne, R., and Baer, Y., ESCA Applied to Free Molecules (North-Holland Publishing Co., Amsterdam 1969).

[17] Mansfield, M. W. D., Ph.D. Thesis, Physics Dept., Imperial College London (1969).

[18] Minnhagen, L., Strihed, H., and Peterson, R., Arkiv. för Fys. Bd $39 \mathrm{nr}$ 34, 471 (1969).

[19] Moore, C. E., Atomic Energy Levels, Nat. Bur. Stand. (U.S.) Circ. 467, Vols. 2 and 3 (1952, 1958). 